\title{
Different Post Placement Strategies for the Restoration of Endodontically Treated Maxillary Premolars with Two Roots: Single Post vs Double Post
}

\author{
Valentina Spicciarelli ${ }^{1}$, Crystal Marruganti ${ }^{2}$, Marco Martignoni ${ }^{3}$, Ludovica Andreatti ${ }^{4}$, Tiziana Doldo ${ }^{5}$, Marco Ferrari ${ }^{6}$, \\ Simone Grandini ${ }^{7}$
}

\begin{abstract}
Aim and objective: The present study compared the fracture strength and failure pattern of endodontically treated, bi-rooted, maxillary premolars with different number of coronal walls and postendodontic restoration (one vs double post).

Materials and methods: 105 premolars were divided into 3 groups according to the number of residual walls: control group (intact teeth; $n$ $=15$ ), group 1 ( 3 residual walls; $n=45$ ), group 2 ( 2 residual walls; $n=45$ ). Each test group was then divided into 3 subgroups ( $n=15$ each) according to postendodontic restoration: no post (A), 1 post (B) or 2 posts (C). A load was applied parallel to the longitudinal axis of the teeth, thus simulating physiological occlusion. ANOVA and Tukey's tests were used to detect fracture strength differences among groups, while Chisquare test was used to check differences in fracture pattern.

Results: No significant differences were observed between control group (intact teeth) and groups A1 ( $p=0.999), \mathrm{B} 1(p=0.997)$ and C1 $(p=1.000)$; statistically significant differences were detected between control group and groups A2 $(p<0.001), \mathrm{B} 2(p<0.001)$ and C2 ( $p<0.05)$. Different post placement techniques were non-significantly associated with fracture pattern in both groups $1(p=0.666)$ and $2(p=0.143)$ while, irrespective of the number of posts, the presence of the post was significantly associated with the fracture pattern in teeth with two residual walls. The double-post technique did not further improve the fracture resistance of hardly damaged endodontically treated maxillary bi-rooted premolars compared to single-post technique. Therefore, the insertion of a single post in the palatal canal could be a safer and more conservative choice.

Clinical significance: The double-post technique did not further improve the fracture resistance of severely structurally compromised endodontically treated maxillary premolars with two roots compared to the single-post technique. Therefore, the safer and less invasive treatment is the placement of a single post in the palatal canal.

Keywords: Double-post, Fracture strength, Laboratory research, Post-endodontic restoration, Single-post.

The Journal of Contemporary Dental Practice (2020): 10.5005/jp-journals-10024-2980
\end{abstract}

\section{INTRODUCTION}

Planning of postendodontic restorations is a controversial topic in literature. Devitalized teeth are more liable to fracture than vital teeth due to either chemical (dentin dehydration) and mechanical (loss of dental structure) characteristics., ${ }^{1,2}$ In fact, the endodontic treatment represents a risk factor for the development of vertical root fractures (VRF) within the first 8 years after treatment, thus increasing the probability of tooth loss. ${ }^{3}$

Premolars are more likely to develop VRF than other teeth due to their anatomical features characterized by steep cuspidal slopes, as well as their position in the dental arch which makes them more subjected to detrimental lateral forces. ${ }^{4}$ The incidence of VFR in premolars ranges from $11.5 \%$ for the upper to $17.5 \%$ for the lower premolars. ${ }^{5}$ Indeed, a retrospective study on 468 teeth that had fractured in vivo demonstrated that $78 \%$ of them were premolars, $62 \%$ of which being maxillary premolars. ${ }^{6}$ In recent years, great attention has been paid to the use of fiber posts in order to reduce the risk of VRF. ${ }^{7}$ Contrary to metal posts, adhesive endocanal posts eliminate the need for over-preparation, as well as being less expensive and time-consuming., 8

Clear guidelines for post placement are still lacking. A discriminant in the decision-making process as to whether or not to place an endocanal post is the amount of residual dental structure: whenever the residual tooth structure is limited and an
1,3,4,7 Department of Medical Biotechnologies, Unit of Endodontics and Restorative Dentistry, University of Siena, Siena, Italy

${ }^{2}$ Department of Medical Biotechnologies, Undergraduate Program in Dentistry, University of Siena, Siena, Italy

${ }^{5}$ Department of Medical Biotechnologies, Unit of Orthodontics, University of Siena, Siena, Italy

${ }^{6}$ Department of Medical Biotechnologies, Unit of Prosthodontics, University of Siena, Siena, Italy

Corresponding Author: Crystal Marruganti, Department of Medical Biotechnologies, Undergraduate Program in Dentistry, University of Siena, Siena, Italy, Phone: +39 0577 585772, e-mail: marruganti@gmail. com

How to cite this article: Spicciarelli V, Marruganti C, Martignoni $M$, et al. Different Post Placement Strategies for the Restoration of Endodontically Treated Maxillary Premolars with Two Roots: Single Post vs Double Post. J Contemp Dent Pract 2020;21(12):1374-1378.

Source of support: Nil

Conflict of interest: None

indirect restoration would not be feasible, placing a post seems to be beneficial. ${ }^{10}$ Endocanal posts have been demonstrated to increase core retention and to distribute forces along the tooth so as to reduce the risk of fracture. ${ }^{11}$ Moreover, fiber post placement was also reported significantly affect fracture resistance of 
endodontically treated bi-rooted maxillary premolars. A recent study ${ }^{12}$ demonstrated that teeth with a single post placed in palatal root showed less fracture strength compared to those restored with two posts placed in both buccal and palatal roots; moreover, this study suggested that placing a post in the palatal canal was preferable, as it maintained the restorability of the tooth.

To our knowledge, literature is still incomplete regarding the influence of number of posts and remaining tooth substance on fracture strength and failure pattern of endodontically treated maxillary premolars with 2 separate roots.

The null hypothesis tested $\left(\mathrm{H}_{0}\right)$ was that there were no statistically significant differences in fracture strength between maxillary premolars with a different amount of residual dental structure restored with one, two or no posts.

\section{Materials and Methods}

\section{Teeth Collection}

One hundred and five maxillary premolars with 2 roots, extracted for periodontal or orthodontic reasons, were collected. Informed consent was obtained from patients for collection of extracted teeth for the use of research purpose. Teeth were cleaned of remaining tissues, using a manual scaler (Hu Friedy, Chicago, II, USA) and an ultrasonic scaler (E.M.S., Nyon, Switzerland) and examined under a $4.5 \times$ stereomicroscope (Nikon SMZ645; Tokyo, Japan) to exclude the presence of any external radicular cracks. The exclusion criteria included caries, fractures, previous endodontic or restorative treatment, and anatomical irregularities. Samples were stored in saline solution $(0.9 \%)$ at a temperature of $37^{\circ} \mathrm{C}$ to prevent dehydration.

The buccolingual and mesiodistal dimensions of each crown, as well as the distance between the cementoenamel junction and the occlusal surface were recorded with a digital caliper (Tchibo, Hamburg, Germany) and used to divide the samples homogeneously into one control group $(n=15)$ and 2 test groups ( $n=45$ each) according to the number of residual walls. Then, each test group was divided into 3 subgroups ( $n=15$ each) according to the number of posts placed (Table 1). Oval-shaped access cavities reflecting the anatomy of the pulp chamber were realized by a single operator. In group 2 samples the mesial wall was removed, whereas both the mesial and distal walls were removed in group 3 samples in order to create MOD cavities (Fig. 1).

\section{Endodontic Treatment}

For all root canals patency was checked with a K-file \#10. Chemomechanical preparation was performed by a single operator using Reciproc system R25 (Dentsply-VDW, Munich, Germany) to the working length. Irrigation was carried out with $5 \mathrm{~mL}$ of $5.25 \% \mathrm{NaOCl}$

Table 1: Control group and test groups according to the number of residual walls, and subgroups according to the number of endocanal posts

\begin{tabular}{lll}
\hline Groups & Residual walls & Post \\
\hline control 0 & $4(n=15)$ & No post \\
Test 1 & $3(n=45)$ & A1: no post \\
& & B1: one post \\
& C1: two posts \\
Test 2 & $2(n=45)$ & A2: no post \\
& & B2: one post \\
& & C2: two posts \\
\hline
\end{tabular}

for 30 minutes during the instrumentation, $2.5 \mathrm{~mL}$ of $17 \%$ EDTA for 2 minutes followed by $2.5 \mathrm{~mL}$ of $5.25 \% \mathrm{NaOCl}$ for 5 minutes as final flush. Canal obturation was achieved through continuous condensation wave technique, the gutta-percha cone was cut leaving $5 \mathrm{~mm}$ of apical seal (BeeFill 2in1, VDW).

\section{Post-endodontic Restoration}

Samples were divided into subgroups. For subgroups A no post was used, for subgroups B and C X-Post ${ }^{\mathrm{TM}}$ No. 1 (Dentsply DeTrey) was used. Root canal obturation was removed with the selected Largo ${ }^{\oplus}$ Peeso Reamer (800-1200 rpm speed); leaving at least $4 \mathrm{~mm}$ of root canal filling in the apical region. Then Easypost ${ }^{\mathrm{TM}}$ Precision Drill corresponding to the selected X-Post (1000-2000 rpm speed) was used to drill the post-space.

Post space was etched for $15 \mathrm{~s}$ using DeTrey Conditioner (Dentsply Sirona, UK) then rinsed for $15 \mathrm{~s}$ and dried with paper points. Prime and Bond NT (Dentsply Caulk, York, PA, USA) adhesive system was used in dual-cure mode, mixed with Self-Cure Activator. Adhesive mix was applied to cavity surface and to post surface and air dried. Core-X flow (Dentsply DeTrey) was placed onto the post surface and to the post hole preparation and stabilized for 20 s. The post-adhesive-resin cement system was light cured for 40 s with an irradiance of $600 \mathrm{~mW} / \mathrm{cm}^{2}$. In subgroups B samples, one post was placed in the palatal root; in subgroups $C$ samples, two posts were placed. Mesial and distal walls were previously restored using a total-etch Adhesive system (XP-Bond, Dentsply srl Italia, Roma, IT) and Ceram-X Duo E2 (Dentsply srl, Rome, IT). In the control group and subgroups $A_{1}, A_{2}$ and $A_{3}$, no posts were used.

\section{Fracture Test}

Samples were embedded in coldcure epoxy resin (Gnathus cold self-curing acrylic resin, Zhermak, Badia Polesine, Italy), leaving an uncovered roots portion of $2 \mathrm{~mm}$ apically from the CEJ. Samples in the resin block were then mounted in a customized holder of a universal testing machine (Triaxal Tester T400 Digital; Controls $\mathrm{Srl}$, Cernusco, Italy) perpendicularly to the horizontal plane. The compressive load was applied on the palatal cusp, $2 \mathrm{~mm}$ from the central fossa, to simulate an occlusal vertical load. The compressive load was applied at a crosshead speed of $1 \mathrm{~mm} /$ minute, until fracture occurred, and the maximum breaking load was recorded in Newton (N). The fracture pattern was classified into either restorable $(\mathrm{R})$, when involving the coronal portion without crossing the CEJ, or unrestorable $(U)$, when involving the root.

\section{Data Analysis}

All statistics were performed using SigmaPlot software for Windows 11.0 (Systat Software, Inc., San Jose, CA, USA). Quantitative variables were expressed as mean (standard deviation) and Confidence Interval (95\%). Normality distribution of data was tested according to Shapiro-Wilk test. The homogeneity of variances was carried out by the Levene's test. One-way analysis of variance (ANOVA) was performed to detect significance between groups. The significance value was set at $p=0.05$. Post hoc Tukey's test was applied for multiple comparisons. Chi-square test was used to check differences between proportions of " $\mathrm{R}$ " (restorable) and " $\mathrm{U}$ " (unrestorable) among groups.

\section{Results}

Levene's test demonstrated the homoscedasticity of the distributions for all groups ( $p=0.824)$. One-way ANOVA test 

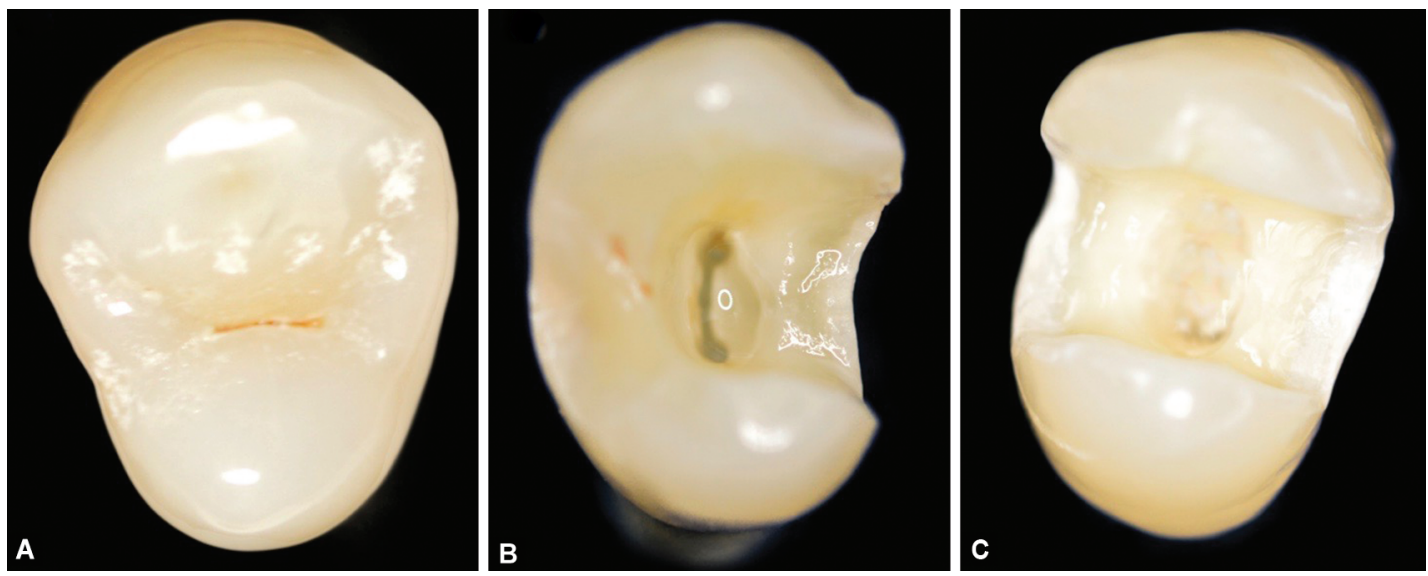

Figs $1 \mathrm{~A}$ to $\mathrm{C}$ : Control group 0 : sound teeth; Test 1: MO cavities; Test 2: MOD cavities

Table 2: Data are presented as mean \pm standard deviation (SD), 95\% confidence interval or percentage

\begin{tabular}{lllll}
\hline & \multirow{3}{*}{$\begin{array}{l}\text { Fracture load } \\
\text { [N] mean }\end{array}$} & & \multicolumn{2}{c}{ Failure mode } \\
\cline { 4 - 5 } Groups & $\pm s d$ & $95 \% \mathrm{Cl}$ & $\begin{array}{l}\text { Restorable } \\
n(\%)\end{array}$ & $\begin{array}{l}\text { Unrestorable } \\
n(\%)\end{array}$ \\
\hline Control 0 & $509.0 \pm 89.5$ & $(467.5 ; 550.5)$ & $11(73 \%)$ & $4(27 \%)$ \\
A1 & $495.2 \pm 70.8$ & $(453.7 ; 536.7)$ & $7(47 \%)$ & $8(53 \%)$ \\
B1 & $491.1 \pm 66.4$ & $(449.6 ; 532.6)$ & $11(73 \%)$ & $4(27 \%)$ \\
C1 & $500.5 \pm 82.8$ & $(459.0 ; 541.9)$ & $12(80 \%)$ & $3(20 \%)$ \\
A2 & $223.9 \pm 89.5$ & $(182.4 ; 265.4)$ & $4(2 \%)$ & $11(73 \%)$ \\
B2 & $345.2 \pm 97.2$ & $(303.7 ; 386.7)$ & $13(87 \%)$ & $2(13 \%)$ \\
C2 & $402.9 \pm 64.7$ & $(361.4 ; 444.4)$ & $14(93 \%)$ & $1(7 \%)$ \\
\hline
\end{tabular}

revealed significant differences between the means of the distributions $(p<0.05)$. Statistics of all groups are shown in Table 2.

No significant differences were observed between control group (intact teeth) and groups A1 ( $p=0.999), \mathrm{B} 1(p=0.997)$ and C1 $(p=1.000)$, while statistically significant differences were detected between control group and groups A2 ( $p<0.001), \mathrm{B} 2(p<0.001)$ and C2 ( $p<0.05)$, respectively. Group 1 obtained significantly higher fracture strength values $(p<0.05)$ compared to the respective samples of group 2.

Group A2 obtained the lowest fracture strength value (223.9 $\pm 89.5 \mathrm{~N}$ ) of all other tested groups. For teeth with two residual walls, the presence of either one post located in the palatal canal $(p=0.002)$ or two posts $(p<0.001)$ significantly increased fracture resistance. No differences were observed between groups B2 and C2 $(p=0.452)$, even though group C2 obtained slightly higher fracture resistance values $(402.9 \pm 64.7 \mathrm{~N})$.

No differences were observed between the different restorative strategies tested in samples with three residual walls. Multiple comparisons results are reported in Table 3.

In $13-27 \%$ of the endodontically treated maxillary premolars restored with a fiber post, catastrophic failure was observed.

Different post placement techniques were non-significantly associated with the fracture pattern of either tooth with three ( $p=0.666)$ and two residual walls $(p=0.143)$; irrespective of the number of posts used, the presence of the post was significantly associated with the fracture mode in teeth with two residual walls (Table 4).
Table 3: Interaction among subgroups $A, B$ and $C$ within the test groups 1 and 2

\begin{tabular}{lllll}
\hline $\begin{array}{l}\text { Difference of } \\
\text { levels }\end{array}$ & $\begin{array}{l}\text { Difference of } \\
\text { means }\end{array}$ & $95 \% \mathrm{Cl}$ & Tvalue & p value \\
\hline A1-0 & -13.7 & $(-102.8 ; 75.3)$ & -0.46 & ${ }^{\S} \mathbf{0 . 9 9 9}$ \\
A2-0 & -285.1 & $(-374.2 ;-196.0)$ & -9.64 & 0.000 \\
B1-0 & -17.9 & $(-107.0 ; 71.2)$ & -0.61 & ${ }^{\S} \mathbf{0 . 9 9 7}$ \\
B2-0 & -163.7 & $(-252.8 ;-74.7)$ & -5.54 & 0.000 \\
C1-0 & -8.5 & $(-97.6 ; 80.6)$ & -0.29 & ${ }^{\S} \mathbf{1 . 0 0 0}$ \\
C2-0 & -106.0 & $(-195.1 ;-17.0)$ & -3.59 & 0.009 \\
A2-A1 & -271.4 & $(-360.4 ;-182.3)$ & -9.18 & 0.000 \\
B1-A1 & -4.2 & $(-93.2 ; 84.9)$ & -0.14 & ${ }_{1} \mathbf{1 . 0 0 0}$ \\
B2-A1 & -150.0 & $(-239.1 ;-60.9)$ & -5.07 & 0.000 \\
C1-A1 & 5.2 & $(-83.8 ; 94.3)$ & 0.18 & $\$ \mathbf{1 . 0 0 0}$ \\
C2-A1 & -92.3 & $(-181.4 ;-3.3)$ & -3.12 & 0.037 \\
B1-A2 & 267.2 & $(178.1 ; 356.3)$ & 9.04 & 0.000 \\
B2-A2 & 121.4 & $(32.3 ; 210.4)$ & 4.10 & 0.002 \\
C1-A2 & 276.6 & $(187.5 ; 365.7)$ & 9.35 & 0.000 \\
C2-A2 & 179.1 & $(90.0 ; 268.1)$ & 6.06 & 0.000 \\
B2-B1 & -145.8 & $(-234.9 ;-56.8)$ & -4.93 & 0.000 \\
C1-B1 & 9.4 & $(-79.7 ; 98.5)$ & 0.32 & ${ }^{\$} \mathbf{1 . 0 0 0}$ \\
C2-B1 & -88.1 & $(-177.2 ; 0.9)$ & -2.98 & ${ }^{\S} \mathbf{0 . 0 5 4}$ \\
C1-B2 & 155.2 & $(66.2 ; 244.3)$ & 5.25 & 0.000 \\
C2-B2 & 57.7 & $(-31.4 ; 146.8)$ & 1.95 & $\S_{0} \mathbf{0 . 4 5 2}$ \\
C2-C1 & -97.5 & $(-186.6 ;-8.5)$ & -3.30 & 0.022 \\
\hline
\end{tabular}

${ }^{\S}$ Indicates no statistically significant differences, while significant differences were detected between all the other interactions $p<0.05$, Tukey's test for multiple comparisons

\section{Discussion}

This ex vivo study assessed the association between the number of posts in maxillary bi-rooted premolars with different levels of coronal structure damage (intact tooth vs loss of 1 vs loss of 2 walls). A statistically significant correlation was detected between the fracture resistance of endodontically treated maxillary first premolars with a different amount of residual dental structure, but no differences were observed between the restoration with one single post located in the palatal canal or two posts, one for each canal: the null hypothesis $\left(\mathrm{H}_{0}\right)$ had to be partially rejected. 
Table 4: When both $p$ values (Pearson and Likelihood ratio) were less than the significance level of 0.05 , a significant difference between the outcome percentage profiles of two groups was reported

\begin{tabular}{llcl}
\hline Difference of levels & & Chi-square & p value \\
\hline A1-A2 & Pearson & 1.292 & 0.256 \\
& Likelihood ratio & 1.304 & 0.253 \\
A1-B1 & Pearson & 2.222 & 0.136 \\
& Likelihood ratio & 2.256 & 0.133 \\
A1-C1 & Pearson & 3.589 & 0.058 \\
& Likelihood ratio & 3.690 & 0.055 \\
B1-C1 & Pearson & 0.186 & 0.666 \\
& Likelihood ratio & 0.187 & 0.666 \\
A2-B2* & Pearson & 10.995 & 0.001 \\
& Likelihood ratio & 11.876 & 0.001 \\
A2-C2* & Pearson & 17.368 & 0.000 \\
& Likelihood ratio & 22.032 & 0.000 \\
B2-C2 & Pearson & 2.143 & 0.143 \\
& Likelihood ratio & 2.916 & 0.088 \\
\hline
\end{tabular}

${ }^{*} p<0.05$, Chi-square test

Maxillary premolars were chosen for this experimental study because they are more prone to vertical fracture than other teeth, due to their anatomical characteristics and their position in the dental $\mathrm{arch} ;{ }^{4}$ moreover, there is lack of evidence about the effectiveness of more than one endocanal post in fracture resistance of maxillary premolars with two separate roots.

One of the major challenges of ex vivo studies is the standardization of the samples due to their anatomical variability, aging and morphological alterations; in the current study, teeth with similar buccolingual and mesiodistal CEJ dimensions and similar root length were selected in order not to affect the results of the experimentation. In test groups, teeth were prepared with standardized extended MO (mesio-occlusal) and MOD (mesialocclusal-distal) cavities, with the aim of simulating the clinical condition of a carious process invading the pulp chamber and therefore requiring an endodontic treatment. In accordance with previous studies, ${ }^{13}$ the amount of residual coronal dental structure appeared to be the most significant prognostic element for endodontically treated maxillary premolars. Single post and two-post techniques did not increase the fracture resistance of teeth that lost one marginal ridge, but a significant increase was detected whenever two marginal ridges were lost. Marginal ridges of at least $2 \mathrm{~mm}$ thickness represent a reinforcing factor and their loss represents a risk factor for tooth fracture. ${ }^{14}$ Cusp elongation in maxillary premolars due to the pulp chamber roof removal while preparing the endodontic access cavity creates a propensity to buccal and palatal cusps separation under occlusal load. ${ }^{15}$ In this study, MOD preparation was considered the simulation of the worst clinical situation; the extended dental removal for MOD cavities realization also influenced the cuspal flexure from polymerization shrinkage of composite restorations, ${ }^{16}$ acting as a preloading, facilitating tooth fracture under occlusal loads. 17,18

Biting force is clinically between 222-445 N for the maxillary premolar area and during clenching the occlusal force is as high as $520-800 \mathrm{~N}$ (average $660 \mathrm{~N}$ ). ${ }^{19,20}$ From the results of our study it was possible to conclude that endodontically treated maxillary premolars that lost both interproximal walls were not able to fully support physiological and para-physiological loads, both in case of single $(345.23 \pm 97.15)$ and two posts $(402.92 \pm 64.65)$ placement. This is in agreement with many authors, who recommended the execution of an indirect restoration in all those cases in which the marginal ridges were lost, in order to guarantee the long-term survival of the tooth. ${ }^{21}$ Therefore, it appears that all the other tested groups in the current study could resist the functional stresses developed in the mouth; however, some clinical circumstances such as thermal or chemical factors, repeated stresses may lead to failure at a point which could be far below the ultimate fracture resistance. Moreover, in the present study an experimental load was applied parallel to the longitudinal axis of teeth in order simulate the physiological occlusion. ${ }^{22-24}$ Since premolars are frequently subjected to lateral forces, this kind of load design may overvalue the fracture strength of tested specimens.

The absence of a fiberglass post most frequently resulted in irreparable fracture patterns. In fact, the group consisting of maxillary premolars with MOD cavities and restored with no fiber post obtained the highest percentage of unfavorable fractures (73\%). Conversely, whenever a post was placed, groups with MOD cavities obtained a higher percentage of favorable fractures $(90 \%)$ than groups that lost one marginal ridge (76\%). The fiber post redistributes stresses in the external surface of the coronal root area; ${ }^{25}$ moreover, the presence of coronal dentinal structure may influence the stress distribution, and, consequently, the failure mode when a post is placed. This condition can be explained by the fact that when massive dental structure loss happens, stresses concentrate mainly in the cervical area, thus causing favorable fracture patterns; however, with the increase of remaining coronal walls, stresses will be increasingly transmitted to the apical area causing irreparable fractures.

From a clinical standpoint, the presence of a post could interfere with the endodontic re-treatment. From the results of this study, in case of maxillary premolars with severely compromised coronal structure, the insertion of two posts has proved to be beneficial; however, it seemed to be insufficient to allow the teeth to withstand physiological and para-physiological stresses. Moreover, the fracture resistance of severely compromised maxillary premolars significantly improved even with the insertion of a single post. In addition, previous studies suggested that only the palatal canal should be used for the post, and the buccal canal should be avoided because of the presence of the buccal furcation groove and a narrow root. ${ }^{26,27}$ The considerable tapering and slender roots, the furcation furrows or developmental hollows on the palatal slope of the buccal root enhance the risk of perforation and fracture both during postspace preparation and re-treatments, ${ }^{28}$ because the mean dentine thickness at the deepest part of the invagination was seen to be too small, around $0.81 \mathrm{~mm}^{29}$

Dentists should consider whether or not to place a post on a case-by-case basis; not every endodontically treated tooth needs an endocanal retention. In maxillary premolars with two roots, the insertion of a single post in the palatal canal could be a safer and more conservative choice and, also, facilitate a possible endodontic re-treatment in the future.

\section{Clinical Significance}

The double-post technique did not further improve the fracture resistance of severely damaged endodontically treated maxillary premolars with two roots compared to the single-post technique. Therefore, the safer and less invasive treatment is the placement of a single post in the palatal canal. 


\section{References}

1. Riviera E, Yamauchi G, Chasldler G. Dentin collagen cross-linked of root filled and normal teeth. J Endod 1988;14:195-199.

2. Ferrari M, Mason PN, Goracci C, et al. Collagen degradation in endodontically treated teeth after clinical function. J Dent Res 2004;83(5):414-419. DOI: 10.1177/154405910408300512.

3. García-Guerrero C, Parra-Junco C, Quijano-Guauque S, et al. Vertical root fractures in endodontically-treated teeth: a retrospective analysis of possible risk factors. J Invest Clin Dent 2017;00:e12273.

4. Schwartz RS, Robbins JW. Post placement and restoration of endodontically treated teeth: a literature review. J Endod 2004;30(5):289-301. DOI: 10.1097/00004770-200405000-00001.

5. Karygianni L, Krengel M, Winter M, et al. Comparative assessment of the incidence of vertical root fractures between conventional versus surgical endodontic retreatment. Clin Oral Investig 2014;18(8):20152021. DOI: $10.1007 / \mathrm{s} 00784-013-1182-1$.

6. Rud J, Omnell K-A. Root fractures due to corrosion diagnostic aspects. Scand J Dent Res 1970;78(5):397-403. DOI: 10.1111/j.1600-0722.1970. tb02088.x.

7. Sorrentino R, Monticelli $F$, Goracci $C$, et al. Effect of residual coronal structure on resistance of teeth restored with posts. G Ital Endod 2012;26:13-21.

8. Grandini S, Goracci C, Tay FR, et al. Clinical evaluation of the use of fiber posts and direct resin restorations for endodontically treated teeth. Int J Prosthodont 2005;18:399-404.

9. Santos Pantaleón D, Valenzuela FM, Morrow BR, et al. Effect of ferrule location with varying heights on fracture resistance and failure mode of restored endodontically treated maxillary incisors. J Prosthodont 2019;28(6):677-683. DOI: 10.1111/jopr.13090.

10. Aurélio IL, Fraga S, Rippe MP, et al. Are posts necessary for the restoration of root filled teeth with limited tissue loss? A structured review of laboratory and clinical studies. Int Endod J 2016;49(9):827835. DOI: 10.1111/iej.12538.

11. Makade CS, Meshram GK, Warhadpande M, et al. A comparative evaluation of fracture resistance of endodontically treated teeth restored with different post core systems: an in-vitro study. J Adv Prosthodont 2011;3(2):90-95. DOI: 10.4047/jap.2011.3.2.90.

12. Sulaiman $\mathrm{E}$, Alarami N, Wong $\mathrm{Yl}$, et al. The effect of fiber post location on fracture resistance of endodontically treated maxillary premolars. Dent Med Probl 2018;55(3):275-279. DOI: 10.17219/dmp/94656.

13. Ibrahim AM, Richards LC, Berekally TL. Effect of remaining tooth structure on the fracture resistance of endodonticallytreated maxillary premolars: an in vitro study. J Prosthet Dent 2016;115(3):290-295. DOI: 10.1016/j.prosdent.2015.08.013.

14. Shahrbaf S, Mirzakouchaki B, Oskoui SS, et al. The effect of marginal ridge thickness on the fracture resistance of endodontically-treated, composite restored maxillary premolars. Oper Dent 2007;32(3):285290. DOI: 10.2341/06-83.

15. Mondelli RF, Ishikiriama SK, de Oliveira Filho O, et al. Fracture resistance of weakened teeth restored with condensable resin with and without cusp coverage. J Appl Oral Sci 2009;17(3):161-165. DOI: 10.1590/S1678-77572009000300006.

16. González López S, Sanz Chinesta MV, Ceballos García L, et al. Influence of cavity type and size of composite restorations on cuspal flexure. Med Oral Patol Oral Cir Bucal 2006;11:536-540.

17. Meredith N, Setchell DJ. In vitro measurement of cuspal strain and displacement in composite restored teeth. J Dent 1997;25(3-4): 331-337. DOI: 10.1016/S0300-5712(96)00047-4.

18. González-López S, Vilchez Díaz MA, de Haro-Gasquet F, et al. Cuspal flexure of teeth with composite restorations subjected to occlusal loading. J Adhes Dent 2007;9:11-15.

19. Hidaka O, Iwasaki M, Saito M, et al. Influence of clenching intensity on bite force balance, occlusal contact area, and average bite pressure. $J$ Dent Res 1999;78(7):1336-1344. DOI: 10.1177/00220345990780070801.

20. Widmalm SE, Ericsson SG. Maximal bite force with centric and eccentric load. J Oral Rehabil 1982;9(5):445-450. DOI: 10.1111/j.13652842.1982.tb01034.x.

21. Soares PV, Santos-Filho PC, Gomide HA, et al. Influence of restorative technique on the biomechanical behavior of endodontically treated maxillary premolars. part II: strain measurement and stress distribution. J Prosthet Dent 2008;99(2):114-122. DOI: 10.1016/S00223913(08)60027-X.

22. Oz FD, Attar N, Sungur DD. The influence of restorative material and glass fiber posts on fracture strength of endodontically treated premolars after extensive structure loss. Niger J Clin Pract 2019;22(6):782-789. DOI: 10.4103/njcp.njcp_11_19.

23. Eapen AM, Amirtharaj LV, Sanjeev K, et al. Fracture resistance of endodontically treated teeth restored with 2 different fiberreinforced composite and 2 conventional composite resin core buildup materials: an in vitro study. J Endod 2017;43(9):1499-1504. DOI: 10.1016/j.joen.2017.03.031.

24. Gokturk H, Sirin KE, Tekin E, et al. The effect of the different restorations on fracture resistance of root-filled premolars. BMC Oral Health 2018;18(1):196. DOI: 10.1186/s12903-018-0663-7.

25. Abduljawad M, Samran A, Kadour J, et al. Effect of fiber posts on the fracture resistance of maxillary central incisors with class III restorations: an in vitro study. J Prosthet Dent 2017;118(1):55-60. DOI: 10.1016/j.prosdent.2016.09.013.

26. Raiden G, Costa L, Koss S, et al. Residual thickness of root in first maxillary premolars with post space preparation. J Endod 1999;25(7):502-505. DOI: 10.1016/S0099-2399(99) 80291-X.

27. Tamse A, Katz A, Pilo R. Furcation groove of buccal root of maxillary first premolars--a morphometric study. J Endod 2000;26(6):359-363. DOI: 10.1097/00004770-200006000-00012.

28. Gutmann JL. The dentine-rot complex: anatomical and biologic considerations in restoring endodontically treated teeth. J Prosthet Dent 1992;67(4):458-467. DOI: 10.1016/0022-3913(92) 90073-J.

29. Hargreaves KM, Cohen S. Cohen's Pathways of the Pulp. 10th ed., St. Louis, MO: Mosby Elsevier; 2011. 148-233. 